\title{
ORIGINAL ARTICLE PDE11A negatively regulates lithium responsivity
}

\author{
G Pathak ${ }^{1}$, MJ Agostino ${ }^{2}$, K Bishara ${ }^{1}$, WR Capell ${ }^{1}$, JL Fisher ${ }^{1}$, S Hegde ${ }^{1}$, BA lbrahim ${ }^{1}$, K Pilarzyk ${ }^{1}$, C Sabin $^{1}$, T Tuczkewycz ${ }^{3,4}$, S Wilson ${ }^{1}$ and
} MP Kelly ${ }^{1}$

Lithium responsivity in patients with bipolar disorder has been genetically associated with Phosphodiesterase 11A (PDE11A), and lithium decreases PDE11A mRNA in induced pluripotent stem cell-derived hippocampal neurons originating from lithium-responsive patients. PDE11 is an enzyme uniquely enriched in the hippocampus that breaks down cyclic AMP and cyclic GMP. Here we determined whether decreasing PDE11A expression is sufficient to increase lithium responsivity in mice. In dorsal hippocampus and ventral hippocampus (VHIPP), lithium-responsive C57BL/6J and 129S6/SvEvTac mice show decreased PDE11A4 protein expression relative to lithium-unresponsive BALB/CJ mice. In VHIPP, C57BL/6J mice also show differences in PDE11A4 compartmentalization relative to BALB/CJ mice. In contrast, neither PDE2A nor PDE10A expression differ among the strains. The compartment-specific differences in PDE11A4 protein expression are explained by a coding single-nucleotide polymorphism (SNP) at amino acid 499, which falls within the GAF-B homodimerization domain. Relative to the BALB/CJ 499T, the C57BL/6J 499A decreases PDE11A4 homodimerization, which removes PDE11A4 from the membrane. Consistent with the observation that lower PDE11A4 expression correlates with better lithium responsiveness, we found that Pde11a knockout mice (KO) given $0.4 \%$ lithium chow for $3+$ weeks exhibit greater lithium responsivity relative to wild-type (WT) littermates in tail suspension, an antidepressant-predictive assay, and amphetamine hyperlocomotion, an anti-manic predictive assay. Reduced PDE11A4 expression may represent a lithium-sensitive pathophysiology, because both C57BL/6J and Pde11a KO mice show increased expression of the pro-inflammatory cytokine interleukin-6 (IL-6) relative to BALB/CJ and PDE11A WT mice, respectively. Our finding that PDE11A4 negatively regulates lithium responsivity in mice suggests that the PDE11A SNPs identified in patients may be functionally relevant.

Molecular Psychiatry (2017) 22, 1714-1724; doi:10.1038/mp.2016.155; published online 20 September 2016

\section{INTRODUCTION}

Lithium is argued to be the best treatment option for patients with bipolar disorder, but it has a narrow therapeutic window due to significant side effect liability. ${ }^{1}$ If we could selectively augment the beneficial mood stabilizing effects of lithium in the brain without increasing its harmful peripheral side effects, we might be able to improve the safety margin of lithium treatment for patients with bipolar disorder because mood stabilization could be achieved with systemically lower doses. One way to achieve this selective augmentation of lithium's positive effects is to target a molecule that is selectively expressed in the brain.

Phosphodiesterase 11A (PDE11A) hydrolyzes cyclic AMP (CAMP) and cyclic GMP (cGMP) equally well ${ }^{2,3}$ and is the only PDE to be preferentially expressed in the hippocampus. ${ }^{4-6}$ We have shown in rodents that PDE11A4, the longest PDE11A isoform, is almost exclusively expressed in CA1 and subiculum of the ventral hippocampus (VHIPP), with minimal expression in the dorsal HIPP (DHIPP) $)^{4,6}$ and little to no protein expression outside of the brain. ${ }^{5}$ Genetic findings in humans suggest a role for PDE11A in brain function $^{7-12}$ (but see Laje et al., ${ }^{13}$ Perlis et al. ${ }^{14}$ ). For example, PDE11A single-nucleotide polymorphisms (SNPs) have been associated with major depression and antidepressant response $^{7-9}$ (but see Laje et al., ${ }^{13}$ Perlis et al. ${ }^{14}$ ). Of particular interest to the present study, PDE11A SNPs have been associated with lithium responsiveness in patients with bipolar disorder, ${ }^{10,11}$ a PDE11A inactivating mutation has been associated with increased suicide risk $^{12}$ - a symptom lithium uniquely reduces in patients with bipolar disorder, ${ }^{15}$ and lithium decreases PDE11A mRNA expression in induced Pluripotent Stem Cell (iPSC)-derived hippocampal neurons originating from lithium-responsive patients but not from lithium-unresponsive patients. ${ }^{16}$

A relationship between lithium and cyclic nucleotide signaling is well precedented. Lithium is known to modulate cyclic nucleotide signaling (cf., Gould et al. ${ }^{17}$ ), and disturbances in cyclic nucleotide signaling have repeatedly been observed in patients with bipolar disorder (for example, refs 18-26). Interestingly, the alterations in cyclic nucleotide signaling identified in patients with bipolar disorder can be subcellular compartment specific, ${ }^{18,23,25}$ as can be the effects of lithium on cyclic nucleotide signaling. ${ }^{27-29}$ Therefore, we determined whether PDE11A expression and/or compartmentalization differ between mouse strains that respond well versus poorly to lithium. Furthermore, we determined whether differences in PDE11A compartmentalization are due to a non-synonymous coding SNP that exists between mouse strains at amino acid 499, which is positioned within the GAF-B homodimerization domain. We also established whether decreasing PDE11A expression is sufficient to strengthen the antidepressant-like effects of lithium in the tail suspension test (TST) and the anti-manic-like effects of lithium in amphetaminestimulated hyperactivity by testing Pde11a wild-type (WT) and knockout (KO) mice. Finally, we determined whether PDE11A controls protein expression of the pro-inflammatory cytokine

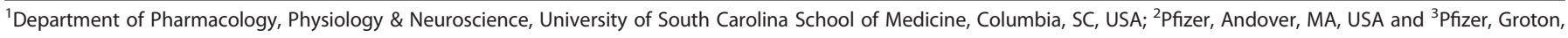

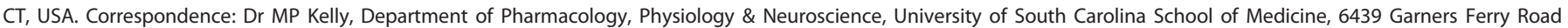
VA Bldg 1, 3rd Floor, D-12, Columbia, SC 29209, USA.

E-mail: Michy.Kelly@uscmed.sc.edu

${ }^{4}$ Current address: Biogen, Boston, MA, USA

Received 11 December 2015; revised 13 July 2016; accepted 18 July 2016; published online 20 September 2016 
interleukin-6 (IL-6), a potential mood disorder biomarker, ${ }^{30-37}$ to clarify whether PDE11A holds promise as a possible target for adjuvant treatment or simply as a patient selection marker.

\section{MATERIALS AND METHODS}

Subjects

C57BL/6J (The Jackson Laboratories (JAX); Bar Harbor, ME, USA), BALB/CJ (JAX) and 129S6/SvEvTac mice (Taconic, Hudson, NY, USA) were ordered in at 10-12 weeks or bred onsite. All C57BL/6J, BALB/CJ and 129S6/SvEvTac mice were group housed and habituated to the colony at least 1 week prior to killing. mRNA analyses and protein analyses of the whole hippocampus using these strains were conducted on cohorts of all males; however, protein analyses on DHIPP vs VHIPP were conducted on mixed cohorts of males and females (cohort used in Figures $1 \mathrm{~d}-\mathrm{g}$ and Supplementary Figure S3 $=50 \%$ female; cohort used in Figure 2, Figures $5 \mathrm{a}-\mathrm{C}$, and Supplementary Figures $\mathrm{S} 2 \mathrm{C}-\mathrm{F}=25 \%$ female). Pde $11 \mathrm{a}$ KO mice were originally developed by Deltagen (San Mateo, CA, USA), and are maintained on a mixed C57BL/6J-C57BL/6N-129S6/SvEvTac background, as previously described. ${ }^{6}$ Pde11a mice used in behavioral experiments were bred at JAX in heterozygous $(H T) \times H T$ matings, with same-sex WT, HT and KO littermates weaned together and shipped onsite at 8-12 weeks old. No more than 2 sets of sex-matched littermates (that is, a WT, HT, KO trio) came from the same litter. Upon delivery, Cohort1 was single housed for consistency with previous reports ${ }^{6}$ and was tested in TST and then amphetamine-stimulated locomotor activity. Cohort2 remained group housed and was tested only in TST. Pde11a mice used in biochemical studies were bred onsite at University of South Carolina in $\mathrm{HT} \times \mathrm{HT}$ matings and were weaned and group housed as described above. In all KO studies, approximately equal numbers of males and females were used. Animals were housed on a 12:12 light:dark cycle and allowed ad libitium access to food and water. See Figure Legends for $n$ 's. Sample sizes were selected based on previous experiences with these assays. Experiments were carried out in accordance with the National Institutes of Health Guide for the Care and Use of Laboratory Animals (Pub 85-23, revised 1996) and were fully approved by the Institutional Animal Care and Use Committees of the University of South Carolina and Pfizer.

\section{Protein sample preparation}

Animals were killed by cervical dislocation and brain regions immediately dissected onto dry ice and stored at $-80^{\circ} \mathrm{C}$. As previously described, ${ }^{4}$ tissue samples and cell lysates were homogenized in ice-cold lysis buffer (20 mM Tris- $\mathrm{HCl}, \mathrm{pH} 7.5 ; 2 \mathrm{~mm} \mathrm{MgCl}$; Thermo Pierce Scientific (Waltham, MA, USA) phosphatase/protease inhibitor tablet) using a probe sonicator. For immunoprecipitation (IP), lysis buffer also contained $0.5 \%$ Tritox X-100/150 mm NaCl, with IP performed using Protein A and Dynabeads as per manufacturer's instructions (Life Technologies, Bedford, MA, USA). For Native PAGE, lysates were additionally nutated for $30 \mathrm{~min}$ at $4{ }^{\circ} \mathrm{C}$ with $0.5 \%$ Triton X-100, treated with $0.1 \mu \mathrm{ml}^{-1}(\mathrm{v} / \mathrm{v})$ Thermo Pierce nuclease for $15 \mathrm{~min}$ at room temperature, and centrifuged at $20000 \mathrm{~g}$ for $30 \mathrm{~min}$ at $4{ }^{\circ} \mathrm{C}$. For fractionation, homogenates were spun at $1000 \mathrm{~g} \times 10 \mathrm{~min}$ at $4{ }^{\circ} \mathrm{C}$ to remove nuclear fractions. Supernatants were then centrifuged at 89000 r.c.f. $\times 10 \mathrm{~min}$ at $4{ }^{\circ} \mathrm{C}$ to yield a cytosolic supernatant fraction. The pellet was washed with a second resuspension, centrifuging and subsequent rinse with ice-cold lysis buffer. The pellet was then sonicated in ice-cold lysis buffer $+0.5 \%$ Triton X-100, nutated for 30 min at $4{ }^{\circ} \mathrm{C}$, and centrifuged at 60000 r.c.f. $\times 30 \mathrm{~min}$ at $4{ }^{\circ} \mathrm{C}$ to yield the soluble membrane supernatant fraction. Samples were stored at $-80^{\circ} \mathrm{C}$ until use. Protein levels were quantified using the DC Protein Assay kit (Bio-Rad, Hercules, CA, USA) as per manufacturer's instructions.

\section{Western blotting}

Lysates were run on either denaturing NuPAGE Novex 4-12\% Bis-Tris or Native PAGE Novex 4-16\% Bis-Tris gels, as per manufacturer's instruction (Life Technologies). Blots were probed overnight at $4{ }^{\circ} \mathrm{C}$ with antibodies recognizing PDE11A (1:500, PD11A-112AP FabGennix-Frisco, TX, USA $\left.{ }^{4-6}\right)$, PDE2A (1:1000, PD2A-101AP FabGennix $\left.{ }^{38}\right)$, PDE10A (1:2500, Enzo Life Science, Farmingdale, NY, USA $\left.{ }^{39}\right)$, actin (1:10,000, A2066 Sigma, St. Louis, MO), IL-6 (1:200, MAB406 R\&D Systems, Minneapolis, MN, USA $\left.{ }^{40}\right)$, GFP (1:2000, sc-8334 Santa Cruz, Dallas, TX, USA), or RFP (1:10 000, A00682 Genscript, Township, NJ, USA). Blots were then processed as previously published. ${ }^{4,6,41}$
In situ hybridization

Animals were killed by cervical dislocation. Brains were cryosectioned at $20 \mu \mathrm{m}$ and autoradiography for the various PDEs was performed as previously reported. ${ }^{4-6,38,42}$ For Ahrgap32 autoradiography was similarly performed using the following antisense probe ( $3^{\prime}$-AGCCCTGTTTGAGTCAGTCTCAGTGAGAGCTCTCTG-5', NM_177379.4), the specificity of which was confirmed by obtaining identical labeling with a second antisense probe sequence and no signal with the sense version of the probes.

\section{Plasmid generation}

Plasmids encoding either mouse Pde11a4 (NM_001081033; 499A) with $\mathrm{N}$-terminally fused $\mathrm{EmGFP}^{43}$ (A206Y mutation introduced to prevent EmGFP dimerization) or an isolated Pde11a4 GAF-B domain (aa388-558 of NP_001074502.1,which includes 14 upstream amino acids as a spacer) with $\mathrm{N}$-terminally fused $m$ Cherry ${ }^{44}$ were synthesized in pUC 57 by Genscript and subsequently subcloned into pcDNA3.1+ mammalian expression vectors (Life Technologies). 499T/499D mutations were generated using the QuikChange procedure and products (Agilent Technologies, Santa Clara, CA, USA). Oligonucleotide primers were synthesized by Integrated DNA Technologies (Coralville, IA, USA) and mutations were verified by DNA sequencing (Functional Biosciences, Madison, WI, USA). Similar constructs expressing only EGFP or mCherry were used as controls.

\section{Cell culture and transfections}

COS-1 and HEK293T cells (ATCC, Manassas, VA, USA) were grown in 24-well plates or $100 \mathrm{~mm}$ dishes containing Glutamax media (Gibco, Gaithersburg, $\mathrm{MD}$, USA) $/ 10 \%$ fetal bovine serum (FBS) at $37^{\circ} \mathrm{C} / 5 \% \mathrm{CO}_{2}$. Plasmid DNA was transiently transfected using Optimem and Lipofectamine 2000 as per manufacturer's instructions (Life Technologies). The morning following transfection, Optimem was replaced with Glutamax media/5\% FBS and cells continued to grow for 5-24 h. Cells were fixed with $4 \%$ paraformaldehyde for imaging studies or harvested in lysis buffer for Westerns. Experiments shown in Figures $3 \mathrm{~d}-0$ were repeated $\geqslant 3$ times with similar results.

\section{Microscopy}

For data collection, cells were imaged using a Leica HC PL Fluotar 10X/ $0.3 \mathrm{c} /$ //D objective on an inverted Leica DMIL microscope. One digital picture per well or dish was collected using NIS-Elements BR-2.30, and an experimenter blind to treatment then categorized the EmGFP-PDE11A4 expression pattern for each labeled cell in the image (cytoplasmic-only vs containing aggregates). Images shown in figure were collected using an LPI anFL PH2 40X/0.65 $\infty / 1.2$ objective on an EVOSfl digital microscope.

\section{Drugs}

Lithium carbonate and control diets were obtained from Harlan Teklad. [0.2\%] and [0.4\%] were selected based on previous work showing [0.4\%] achieves clinically-relevant plasma exposures. ${ }^{46}$ Mice were fed the chow for $3+$ weeks and given access to $0.9 \%$ saline to mitigate potential ion imbalances. ${ }^{46}$ A $1.78 \mathrm{mg} / \mathrm{kg}$ subcutaneous dose of D-amphetamine (Sigma-Aldrich, salt corrected and dissolved in sterile saline at $10 \mathrm{ml} \mathrm{kg}^{-1}$ ) was selected based on our previous experience. ${ }^{41,47}$

\section{Behavior}

To conduct TST, Scotch tape was wrapped around the mouse's tail and then affixed to the hook of a Hamilton-Kinder TS100 tail suspension system. The time spent immobile during a 6-min trial was automatically recorded. ${ }^{48,49}$ Locomotor activity was recorded using the Accuscan Versamax system and Versadat software as previously described. ${ }^{6,41,47}$

\section{Data analyses}

Data were collected in a blinded fashion, with technical variables counterbalanced across biological variables, and analyzed using Statistica 9.0 (Tulsa, OK, USA) or Sigmaplot 11.1 (San Jose, CA, USA). As previously described, ${ }^{6,41,42}$ to mitigate non-specific effects related to transfer efficiencies, film exposures, etc. across gels, Western blot data on a given gel were normalized to a reference group (for example, WT). Data were analyzed by analysis of variance, repeated measure analysis of variance, Student's $t$-test $(t)$, Mann-Whitney rank sum $(T)$, Wilcoxon signed-rank test $(Z)$ or Pearson's product moment correlation as appropriate. $T$-tests were two sided. NOTE: where parametric tests failed equal variance or normality, 

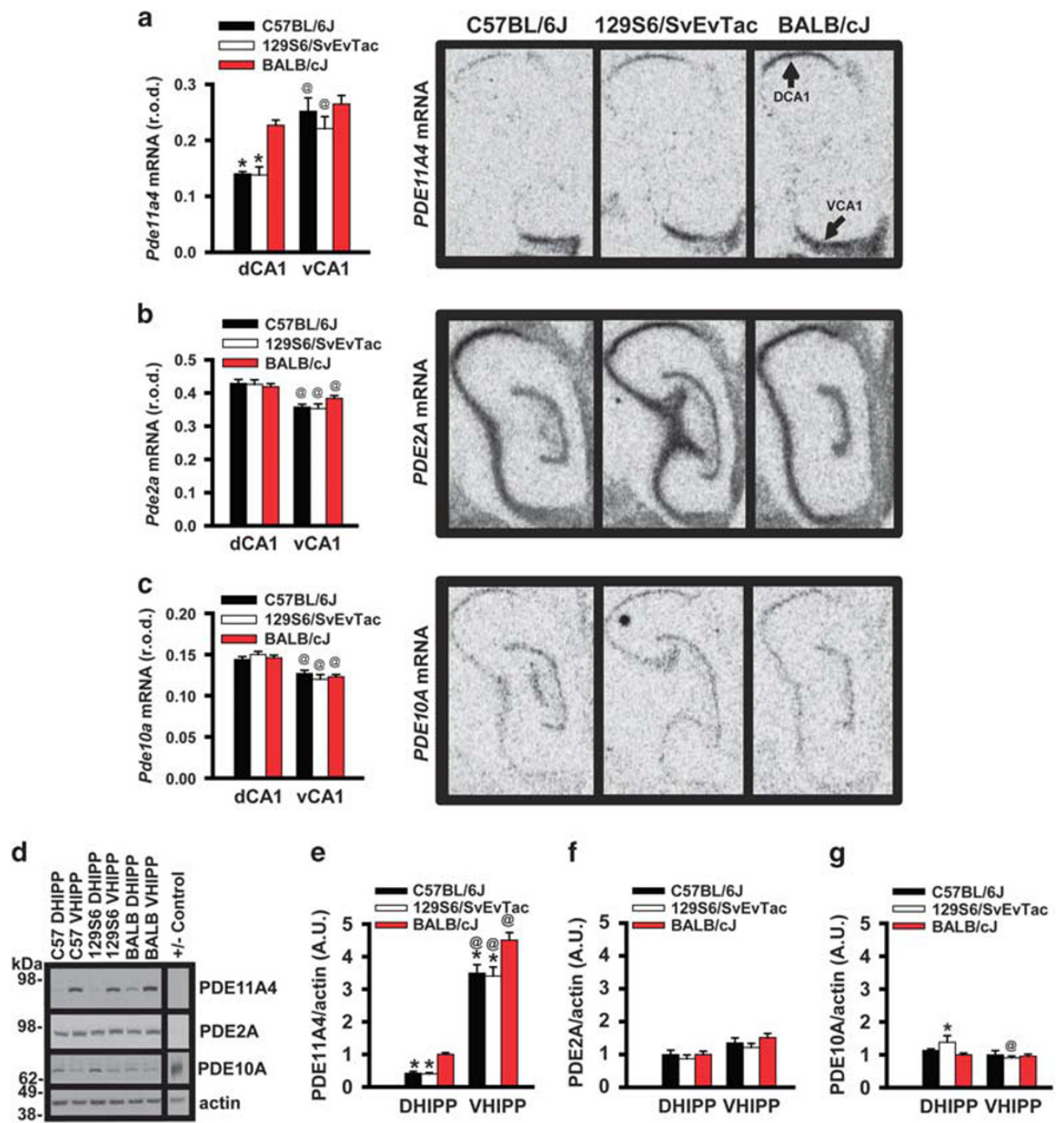

Figure 1. C57BL/6J mice express significantly lower levels of PDE11A4 mRNA and protein than do BALB/cJ mice. To determine whether lithium responsiveness may correlate with PDE11A expression across mouse strains, we measured PDE11A4 expression in hippocampi taken from mice that respond well (C57BL/6J), moderately well (129S6/SvEvTac) or poorly to chronic lithium (BALB/CJ). (a) PDE11A4 mRNA expression in the C57BL/6J $(n=7)$ and 129S6/SvEvTac mice $(n=7)$ is significantly lower than that in the BALB/cJ mice $(n=6)$; however, this difference in mRNA expression is restricted to dorsal CA1 (DCA1). Data is shown for the oligonucleotide probe that selectively recognizes the PDE11A4 isoform, but is representative of results obtained with 3 different oligonucleotide probes (see Supplementary Figure S1 for data collected in a second cohort). Surprisingly, BALB/CJ mice fail to show the typical enrichment of PDE11A4 mRNA expression in ventral CA1 (VCA1) vs DCA1, as has been previously reported in mice and rats and can be seen here in the C57BL/6J mice and 129S6/SvEvTac mice. (b) The expression of PDE2A and (c) PDE10A mRNA are not significantly different between strains, suggesting specificity of the PDE11A4 effect. (d) Western blots measuring PDE11A4 (- control=PDE11A KO VHIPP), PDE2A ( - control=cerebellum), PDE10A (+ control=striatum), and actin. (e) C57BL/6J and 129S6/SvEvTac mice express significantly less PDE11A4 protein relative to BALB/cJ mice in both DHIPP and VHIPP ( $n=12$ per strain). Further, all 3 strains demonstrate far more expression of PDE11A4 protein in VHIPP vs DHIPP, despite the lack of difference at the mRNA level in the BALB/cJ mice. These effects were replicated in a second cohort of mice (Supplementary Figure S2). (f) Neither PDE2A nor (g) PDE10A protein expression differ between the strains, again suggesting specificity of the PDE11A4 effect. Post hoc, ${ }^{*}$ vs BALB/cJ, $P<0.001 ;{ }^{\circledR} \mathrm{Vs} D C A 1$ or DHIPP, $P<0.017-0.001$. Data pass normality and equal variance and are graphed as means \pm s.e.m. Brightness and contrast adjusted for graphical clarity of autoradiograph and Western blot images. a.u., arbitrary units; DHIPP, dorsal hippocampus; PDE11A, phosphodieserase 11A; PDE2A, phosphodiesterase 2A; PDE10A, phosphodiesterase 10A; R.O.D., relative optical density; VHIPP, ventral hippocampus.

nonparametric tests were used. Data were analyzed for effect of strain, brain region, fraction, plasmid, genotype, and/or sex where n's were sufficient to examine a potential effect of sex (that is, if $n>8$ per sex per group). In cases of significant analysis of variances, post hoc analyses were conducted using the Student-Newman-Keuls method. As previously described, ${ }^{6,41,42,50}$ statistical outliers $>2$ standard deviations from the mean were removed from analyses (Figure $2 \mathrm{~b}, 1$ of 16 subjects; Figures $3 \mathrm{k}$, 1 of 72 samples; Figure 3n, 1 of 15 data points; Figure 3o, 2 of 22 data points; Figure $4 a, 1$ of 36 data points; Figure $4 c, 1$ of 77 subjects; Figure $5 b$, 2 out of 32 data points; Figure $5 c$, 1 out of 32 data points; Figure 5e, 2 of 36 subjects). Significance was defined as $P<0.05$.

\section{RESULTS}

C57BL/6J mice, which respond well to lithium, express significantly lower levels of PDE11A4 than do BALB/cJ mice, which respond poorly to lithium

PDE11A4 expression and compartmentalization were measured in the hippocampi of mice that respond well (C57BL/6J), moderately (129S6), or poorly to chronic lithium (BALB/CJ). ${ }^{46,53}$ Pde 11 a4 mRNA expression is significantly lower in the C57BL/6J and 129S6/ SvEvTac mice relative to the BALB/cJ mice, but only in dorsal (d) 

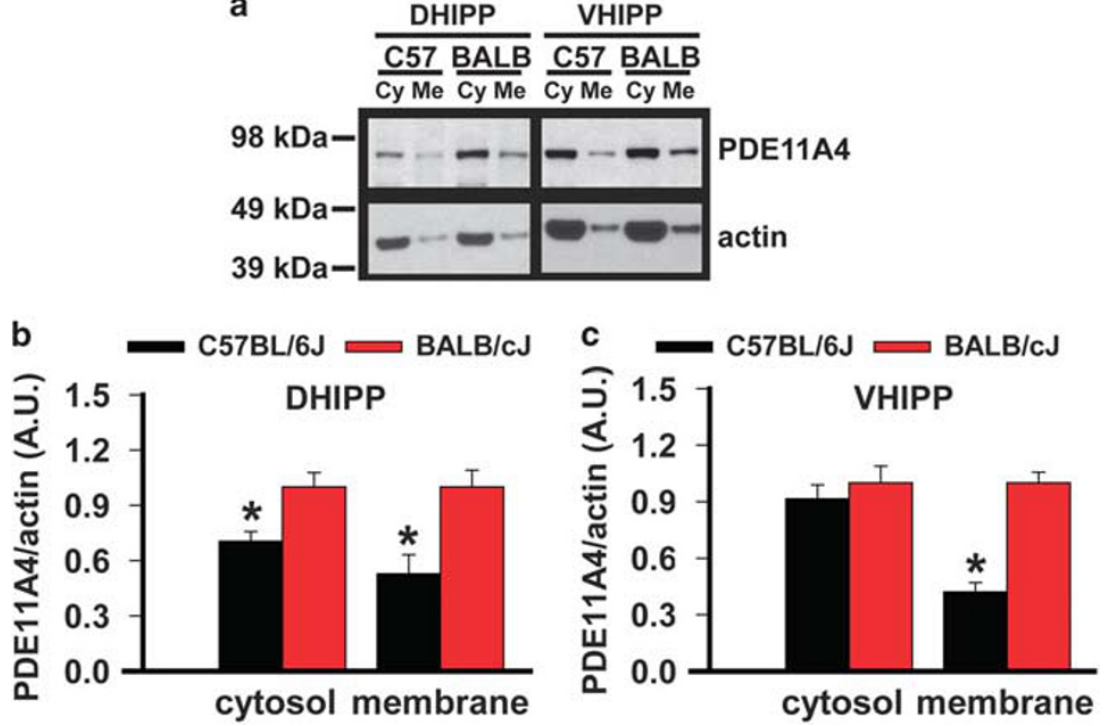

Figure 2. $\mathrm{C} 57 \mathrm{BL} / 6 \mathrm{~J}$ mice compartmentalize PDE11A4 protein in VHIPP differently than do BALB/CJ mice. To determine whether PDE11A4 protein expression differences between $\mathrm{C} 57 \mathrm{BL} / 6 \mathrm{~J}$ and $\mathrm{BALB} / \mathrm{cJ}$ mice might be specific to select subcellular compartments, DHIPP and VHIPP samples were biochemically fractionated and then (a) probed by Western blot. (b) C57BL/6J mice showed lower PDE11A4 protein expression relative to BALB/cJ mice in both the cytosolic and membrane compartments of the DHIPP, (c) but only in the membrane compartment of the VHIPP. This effect was replicated in a second cohort of mice (Supplementary Figure S3). DHIPP, $n=6$ per strain for membrane and 8 per strain for cytosol; VHIPP, $n=8$ per strain per compartment. Post hoc, ${ }^{*}$ vs BALB/cJ, $P<0.01-0.001$, "vs cytosol, $P<0.001$. Data sets passed normality and equal variance and are graphed as means \pm s.e.m. Brightness and contrast adjusted for graphical clarity of blot images. DHIPP, dorsal hippocampus; PDE11A, phosphodiesterase 11A; VHIPP, ventral hippocampus.

and not ventral (v) CA1 (Figure 1a; $F(2,17)=7.15, P=0.006)$. These differences in Pde11a4 mRNA expression were confirmed in a $2^{\text {nd }}$ cohort of mice (Supplementary Figure S1). Similarly, C57BL/6J and 129S6/SvEvTac mice express significantly less PDE11A4 protein relative to BALB/CJ mice in both DHIPP and VHIPP (Figures 1d-g; $F(2,33)=12.29, \quad P<0.001)$, despite the lack of difference in Pde11a4 mRNA expression between the strains in VHIPP (Figure 1a, Supplementary Figure S1A-B). Furthermore, all three strains demonstrate far more expression PDE11A4 protein in VHIPP vs DHIPP (Figures 1d-g; $F(1,33)=622.65, P<0.001$ ), despite the fact that $B A L B / c J$ mice fail to show the prototypical 2-fold enrichment of Pde11a4 mRNA in vCA1 vs dCA $1^{4-6}$ (Figure $1 \mathrm{a}$, Supplementary Figure S1A-B). These differences in PDE11A4 protein expression were confirmed in a second cohort of mice (Supplementary Figure S2). In DHIPP, C57BL/6J mice show lower PDE11A4 protein expression relative to $B A L B / C J$ mice in both the cytosolic and membrane compartments (Figure 2; $F(1,9)=11.13$, $P=0.005)$. Interestingly, in VHIPP, C57BL/6J mice exhibit lower $\mathrm{PDE} 11 \mathrm{~A} 4$ protein expression relative to the $\mathrm{BALB} / \mathrm{cJ}$ mice only in the membrane (Figure 2c; $F(1,14)=10.63, P=0.006$ ). These compartment-specific differences in PDE11A4 protein expression were confirmed in a second cohort of mice (Supplementary Figure S3). The substantial effect of mouse strain on PDE11A4 expression appears to be specific, because expression of PDE2A and PDE10A, the closest related PDEs with appreciable expression in the hippocampus, does not differ across these mouse strains (Figure 1 and Supplementary Figure S1-S2).

C57BL/6J mice compartmentalize PDE11A4 in VHIPP differently than do BALB/CJ mice due to a non-synonymous coding SNP in the GAF-B homodimerization domain

To determine whether genetic differences in the Pde11a sequence might drive the differential compartmentalization of PDE11A4 in VHIPP, we examined the mouse genome and SNPs using the UCSC Genome Browser ${ }^{54}$ and the Jackson Laboratory Phenome
Database (JLPD).$^{55}$ First we determined if the C57BL/6J Pde11a gene demonstrated a SNP homologous to human rs7585543, which was linked to lithium responsiveness in patients with bipolar disorder. ${ }^{10,11}$ The text 'rs7585543' was used as a query in the UCSC Genome Browser (genome.ucsc.edu) and the Multiz genomic alignments of 44 vertebrates were examined closely. Although sequences of many species align well in this region, 600 nucleotides of mouse sequence are absent. Next, SNPs between 29 mouse strains were identified in the JLPD using "Pde11a" as a text query. Only four SNPs differed between $\mathrm{C} 57 \mathrm{BL} / 6 \mathrm{~J}$ and BALB/CJ (129S6/SvEvTac not included in database): three synonymous (rs27939960, rs27939959, rs27981607) and one non-synonymous (rs27963339). The rs27963339 polymorphism encodes an alanine at position 499 in C57BL/6J and a threonine in BALB/CJ. Human PDE11A4 encodes an alanine at position 499, with only 1 variation (glycine; GCA $\rightarrow$ GGA) noted in 121,412 chromosomes sequenced (http://www.ncbi.nlm.nih.gov/SNP/snp_ref.cgi?locusld = 50940, accessed 04/19/16). ${ }^{56}$

Although non-coding SNPs can have functional consequences, ${ }^{57}$ the coding SNP was most likely to yield a differential compartmentalization signal because the SNP (1) leads to a nonphosphorylatable versus a phosphorylatable residue and (2) falls within the PDE11A4 GAF-B homodimerization domain. ${ }^{58}$ Posttranslational modifications and protein-protein binding are the 2 key mechanisms known to regulate PDE trafficking. ${ }^{39,59,60}$ As such, we determined whether 499A vs 499T would differentially affect PDE11A4 homodimerization and if that, in turn, might lead to a differential compartmentalization of PDE11A4.

COS-1 cells were transiently transfected with plasmids containing EmGFP-PDE11A4 encoding the C57BL/6J 499A, the BALB/CJ 499T, or a phosphomimic 499D (Figure 3a). Results were compared to those obtained when PDE11A4 homodimerization was disrupted by co-expressing a dominant-negative isolated GAF-B domain (Figures $3 \mathrm{~b}$ and $\mathrm{c}$ ). Disrupting homodimerization with the isolated GAF-B domain significantly decreases expression of full-length PDE11A4 while generating an $\sim 80 \mathrm{kDa}$ proteolytic 
fragment (Supplementary Figure S4). Thus, total PDE11A4 protein expression was equilibrated between the control mCherry and GAF-B treatments by adding twice the transfection reaction to the
GAF-B treated cells (Supplementary Figure S4A). Although 499T and 499D do not consistently differ from 499A in terms of total PDE11A4 protein expression (Supplementary Figure S4A), they do a

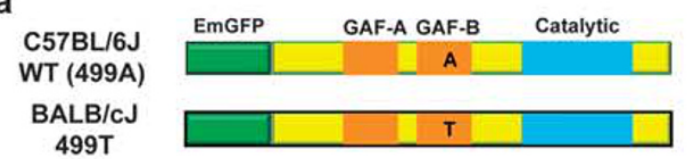

Phosphomimic 499D

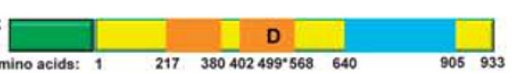

b

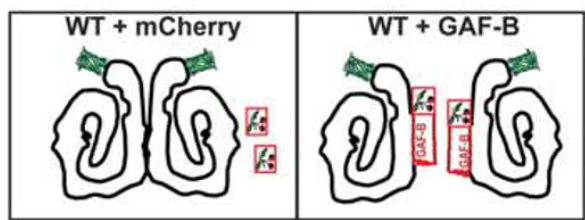

C

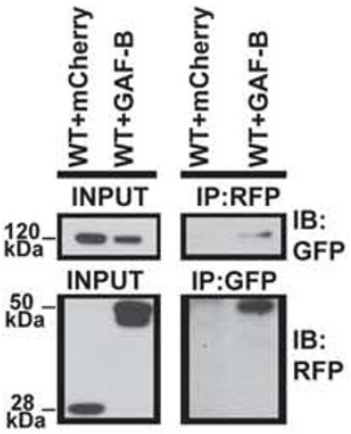

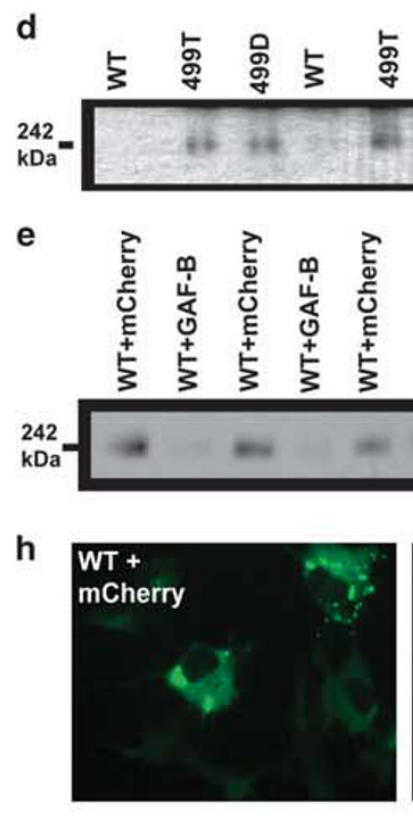

i
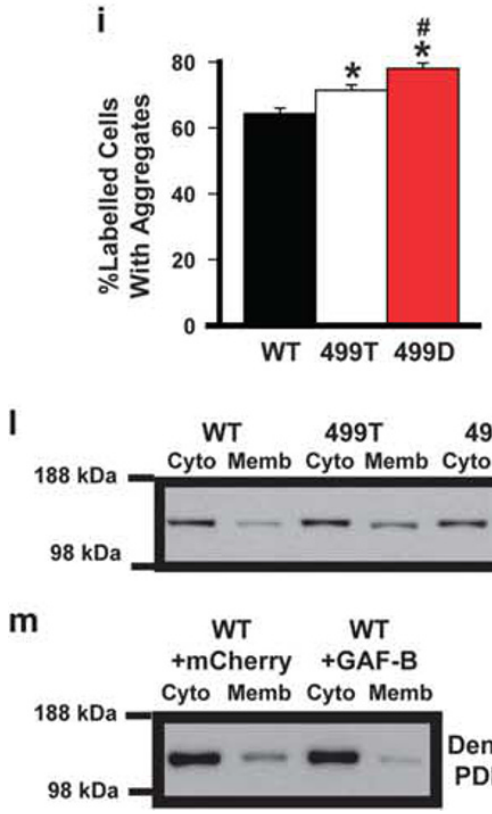

Denatured PDE11A4

j

499D

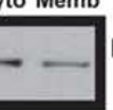

$\mathbf{f}$
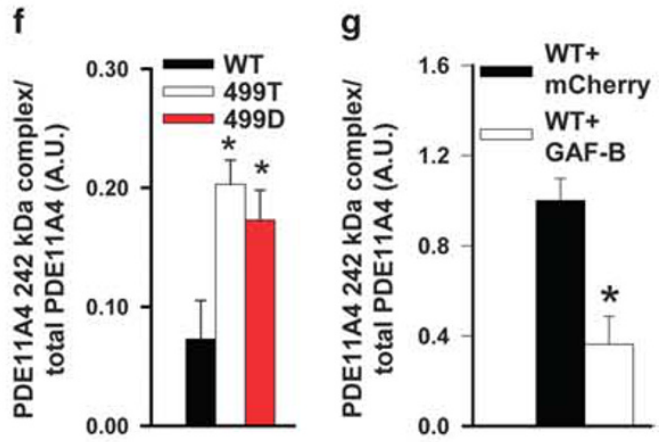

\section{Native}
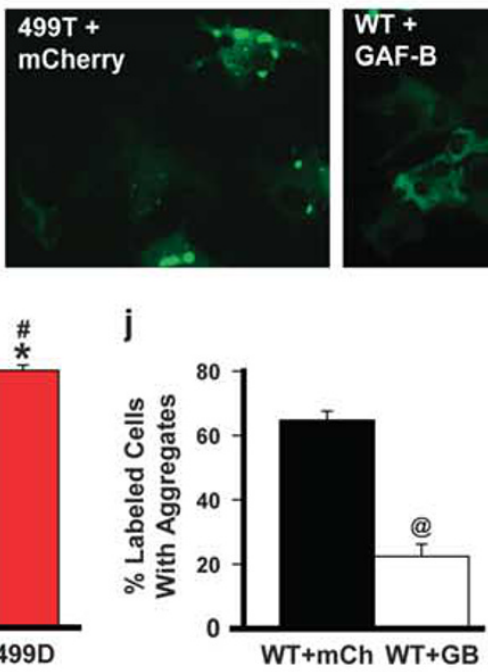

Denatured PDE11A4
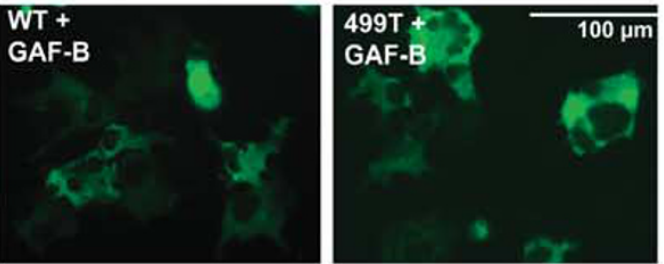

k
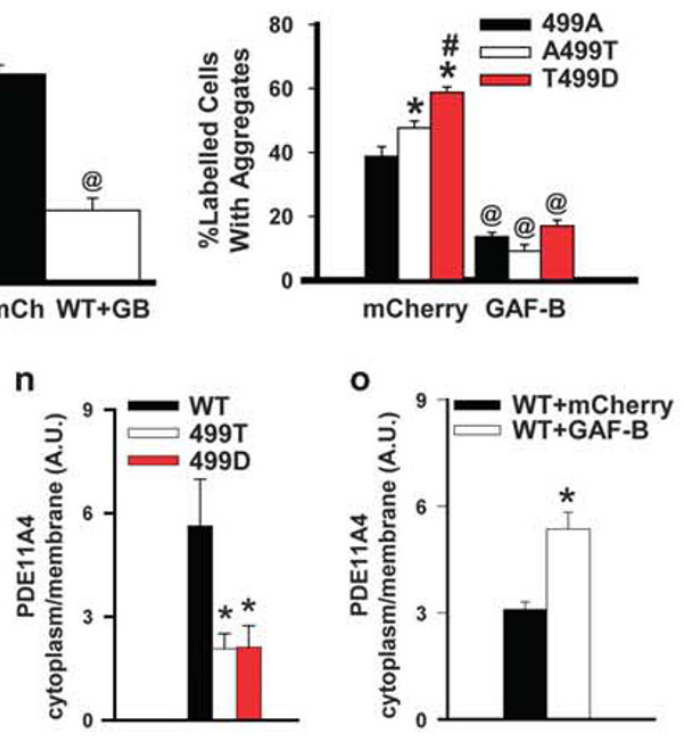
consistently differ in the expression of specific PDE11A4 macromolecular complexes, as determined by Native PAGE. Relative to 499A, 499T and 499D increase expression of a $242 \mathrm{kDa}$ PDE11 A4 complex (Figures $3 d$ and $f ; F(2,8)=6.835, P=0.019$ ), a molecular weight consistent with an EmGFP-PDE11A4 homodimer. Indeed, disruption of homodimerization with the isolated GAF-B domain decreases expression of the $\sim 242 \mathrm{kDa}$ complex (Figures $3 \mathrm{e}$ and g; $t(10)=4.041, P=0.002$ ).

Consistent with these effects on homodimerization, fluorescent microscopy reveals that $499 \mathrm{~T}$ and $499 \mathrm{D}$ promote trafficking of PDE11A4 into aggregates in COS-1 cells $(F(2,21)=16.97$, $P<0.001)$; whereas, the isolated GAF-B domain prevents aggregation (Figures $3 \mathrm{~h}-\mathrm{j} ; t(10)=8.88, P<0.001$ ). 499T and 499D increase the percentage of cells that developed aggregates as opposed to increasing the total number of aggregates per cell or the average size of the aggregates. The ability of 499T and 499D to increase aggregation and the ability of the isolated GAF-B domain to decrease aggregation are also observed in HEK293T cells (499: $F$ $(2,21)=38.95, P<0.001$; GAF-B: $\mathrm{t}(6)=4.20, P=0.006)$. Importantly, adding the isolated GAF-B domain to 499T and 499D completely blocks their ability to promote PDE11A4 aggregation in COS-1 cells (Figures 3h and k), further suggesting that 499T and 499D increase trafficking of PDE11A4 into aggregates by promoting homodimerization. Biochemical fractionations show that 499T and 499D shift PDE11A4 from the cytosol to the membrane relative to $499 \mathrm{~A}$ (Figures $3 \mathrm{l}$ and $\mathrm{n} ; \mathrm{F}(2,41)=8.80, P<0.001$ ), directly mimicking PDE11A4 in VHIPP of C57BL/6J vs BALB/CJ mice (Figure 2c). Furthermore, disrupting homodimerization with the isolated GAF-B domain shifts PDE11A4 in the opposite direction-from the membrane to the cytosol (Figures $3 \mathrm{~m}$ and $\mathrm{o} ; t(18)=4.87$, $P<0.001)$.

PDE11A KO mice exhibit alterations in CAMP signaling and are more sensitive to the antidepressant and anti-manic effects of lithium

As noted above, disturbances in cyclic nucleotide signaling have been repeatedly observed in patients with bipolar disorder (for example, refs 18-26). Direct measures of baseline cAMP signaling in the hippocampus of lithium-responsive versus non-responsive patients is lacking; however, increased basal phosphorylation of CAMP response element binding protein (PCREB) expression has been noted in lymphoblasts of lithium-responsive patients. ${ }^{24}$ As such, we measured pCREB in PDE11A KO vs WT littermates along with expression of an mRNA under the control of pCREB signaling (that is, Arhgap32). We did not observe a significant difference in pCREB phosphorylation between PDE11A KO mice relative to WT littermates when examining the VHIPP as a whole (WT, $1.00 \pm 0.07$ a.u.; KO, $1.132 \pm 0.08$ a.u.; $n=18$ per genotype), likely due to the fact that PDE11A4 is only expressed within a subpopulation of neurons in CA1 and the subiculum. ${ }^{61}$ We were, however, able to detect a significant decrease in Arhgap32 mRNA when measuring expression in VCA1 specifically (Figures $4 a ; t(15)=2.67, P=0.017$ ), a change that is consistent with a localized increase in pCREB phosphorylation. ${ }^{51,52}$

The ability of a drug to reduce immobility in TST is predictive of antidepressant-like activity, ${ }^{62}$ and the ability of a drug to decrease the hyperlocomotor effects of D-amphetamine is indicative of antimanic or antipsychotic-like effects. ${ }^{63,64}$ As we previously reported using single-housed mice, ${ }^{6}$ group-housed PDE11A KO mice show no basal difference in TST immobility relative to WT mice when fed a control diet. Group-housed PDE11A KO mice do, however, exhibit decreased TST immobility in response to a $0.4 \%$ lithium chow while group-housed WT mice do not (Figure 4b; $F(2,49)=3.52, P=0.037)$. Interestingly, when the colony is singlehoused, all genotypes demonstrate a significant reduction in TST immobility in response to $0.4 \%$ lithium chow $(F(2,172)=9.41$, $P=0.003$ ), possibly because single housing decreases PDE11A4 expression in WT mice. ${ }^{65}$ As previously reported, ${ }^{6}$ we found no effect of genotype on D-amphetamine-stimulated hyperactivity when mice consumed a control chow diet (data not shown). When fed $0.4 \%$ lithium chow, however, PDE11A HT and KO mice demonstrate a significantly blunted hyperlocomotor response to D-amphetamine relative to WT littermates during the 70-min $(\mathrm{F}(2,70)=4.50, P=0.015), 75-\mathrm{min}(\mathrm{F}(2,70)=6.76, P=0.002), 80-\mathrm{min}$ $(\mathrm{F}(2,70)=5.64, P=0.005), 85-\mathrm{min}(\mathrm{F}(2,70)=5.12, P=0.008)$ and $90-$ min time bins $(F(2,70)=6.69, P=0.002)$. Altogether, these data show that decreased PDE11A expression is sufficient to increase lithium responsivity.

\section{Lower PDE11A4 expression increases IL-6 expression}

Finally, we determined if PDE11A4 regulates expression of the pro-inflammatory cytokine IL-6, a potential mood disorder biomarker $^{30-37}$ whose elevated expression is reversed by lithium. ${ }^{35}$ Relative to the lithium-unresponsive BALB/CJ mice, the lithium-responsive $\mathrm{C} 57 \mathrm{BL} / 6 \mathrm{~J}$ mice express significantly higher levels of IL-6 in both cytosol $(t(14)=94, P=0.005)$ and membrane

Figure 3. A non-synonymous coding SNP in the PDE11A4 GAF-B domain promotes PDE11A4 homodimerization and alters PDE11A4 compartmentalization. (a) The PDE11A rs27963339 polymorphism affecting amino acid 499 results in an alanine (499A) in C57BL/6J mice (referred to as wild-type (WT) as this is the sequence published in NCBI) and a threonine (499T) in BALB/cJ mice. COS-1 cells were transiently transfected with plasmids encoding an EmGFP-PDE11A4 fusion protein containing a WT 499A, 499T, or 499D (i.e., a phosphomimic aspartate). (b) Alternatively, WT PDE11A4 was co-transfected with either mCherry alone or an isolated GAF-B domain fused to mCherry. Hypothetical structure of PDE11A4 based on. ${ }^{45}$ (c) Co-immunoprecipitation shows that the isolated GAF-B domain binds full-length PDE11A4, enabling the isolated GAF-B domain to act as a dominant-negative that prevents homodimerization. (d, f) Native PAGE shows that the 499T and 499D mutations increase expression of the presumed homodimer at $242 \mathrm{kDa}$ ( $n=4$ per group); whereas, (e, g) expression of the isolated GAF-B domain decreases expression of the presumed homodimer ( $n=6$ per group). Note: The difference in intensity between the WT vs WT$\mathrm{mCherry}$ groups is not related to the co-expression of mCherry but rather reflects the fact that the experiments were conducted separately and different film exposures were necessary for each experiment in order to keep each group of samples within the linear range of the film (e.g., if WT and WT-mCherry exposures were kept constant between experiments, then the 499 groups would be overexposed). (h, i) Consistent with these opposing effects on homodimerization, 499T and 499D promote aggregation of PDE11A4 in COS- 1 cells ( $n=8$ per group); whereas, (j) the isolated GAF-B domain decreases aggregation ( $n=6$ per group). (k) Importantly, the ability of 499T and 499D to promote PDE11A4 aggregation is blocked when homodimerization is prevented by the isolated GAF-B domain ( $n=8$ per group), which suggests the ability of 499T and 499D to increase homodimerization is directly related to their ability to promote the trafficking of PDE11A4 into aggregates. (I, n) Biochemical fractionations show that 499T and 499D shift PDE11A4 from the cytosol (cyto) to the membrane (memb) relative to WT PDE11A4 ( $n=5$ per group), mimicking PDE11A4 compartmentalization in the VHIPP of BALB/cJ vs C57BL/6J mice. (m, o) In contrast, disrupting homodimerization with the isolated GAF-B domain shifts PDE11A4 from the membrane to the cytosol (mCherry, $n=13$; GAF-B, $n=9$ ). The fact that 499T and 499D behave similarly suggests the 499T construct is naturally phosphorylated in COS-1 cells. Post hoc, ${ }^{*}$ vs WT, $P<0.05-0.001$; ${ }^{*}$ vs $499 T, P=0.037-0.011 ;{ }^{\circledR}$ vs mCherry, $P<0.001$. Note that all $n$ 's reflect biological replicates. Data passed normality and equal variance and are graphed as means \pm s.e.m. Brightness and contrast adjusted for graphical clarity of images. a.u., arbitrary units; PDE11A, phosphodiesterase $11 \mathrm{~A}$; SNP, single-nucleotide polymorphism. 

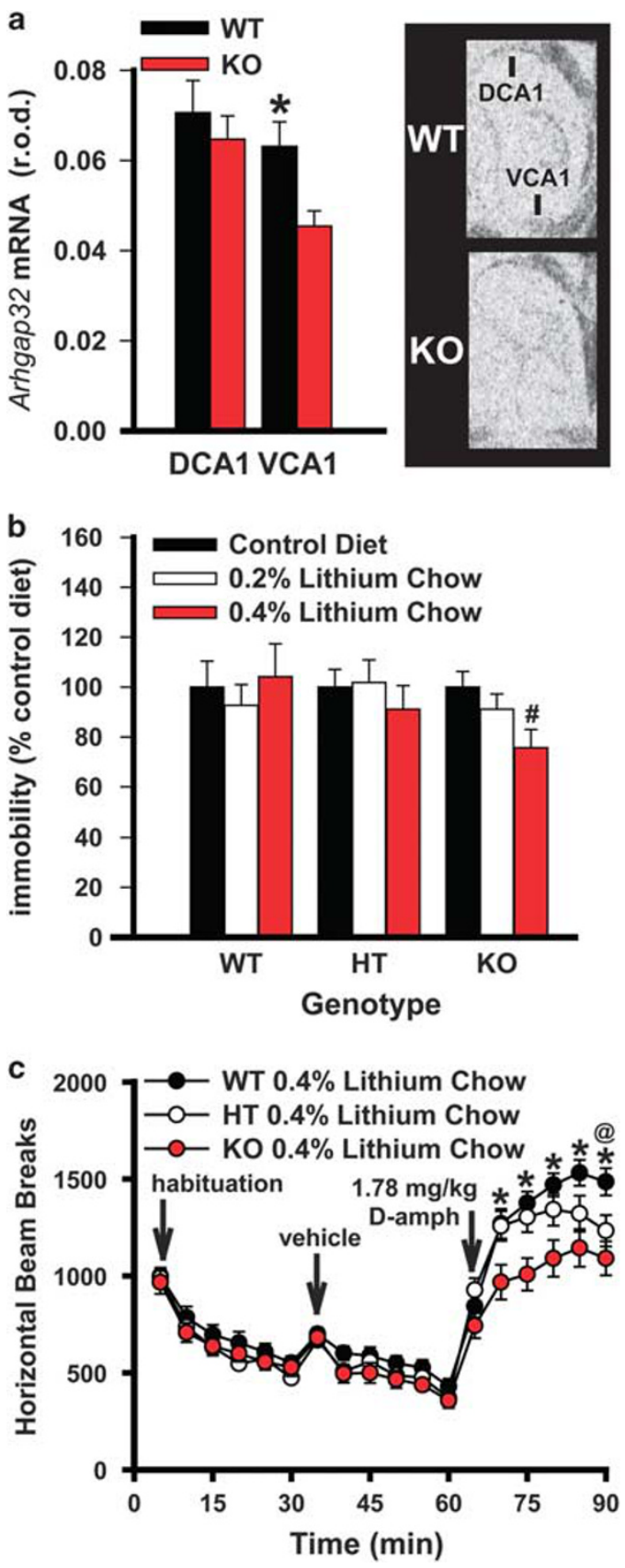

of DHIPP $(t(13)=7.43, P<0.001))$ but only in cytosol of VHIPP $(T(13)=76.00, P=0.001$; Figures 5a-c). In VHIPP of C57BL/6J and $\mathrm{BALB} / \mathrm{CJ}$ mice, lower PDE11A4 expression in membrane correlates with higher IL-6 expression in cytosol (Figure 5d). In DHIPP, PDE11A KO mice demonstrate significantly higher IL-6 expression than do their paired sex-matched WT littermates (Figure $5 \mathrm{e}$; $Z(32)=2.84, P=0.003)$.

\section{DISCUSSION}

Here we show that PDE11A, an enzyme genetically associated with lithium responsivity in patients with bipolar disorder, negatively regulates lithium responsivity in mice. PDE11A4 expression in the DHIPP and VHIPP are significantly reduced in lithium-responsive C57BL/6J and 129S6/SvEvTac mice vs lithiumunresponsive BALB/CJ mice (Figures 1 and 2, Supplementary

Figure 4. Genetic deletion of PDE11A in mice triggers alterations in the CAMP signaling pathway and increases sensitivity to the antidepressant and anti-manic effects of lithium. (a) Autoradiographic in situ hybridization shows that PDE11A KO mice exhibit a significant reduction in Arhgap32 mRNA specifically within ventral CA1 (VCA1) relative to wild-type (WT) littermates $(n=9$ per genotype), indicative of localized increases in pCREB signaling. ${ }^{51,52}$ (b) PDE11A KO mice chronically fed $0.4 \%$ lithium chow $(n=15)$, but not $0.2 \%$ lithium chow $(n=21)$ demonstrated a significant antidepressant-like reduction in tail suspension test (TST) immobility relative to PDE11A KO mice fed a control diet $(n=19)$. PDE11A WT and HT mice showed no significant change in immobility when fed either $0.4 \%$ (WT, $n=16$; HT, $n=16$ ) or $0.2 \%$ lithium chow (WT, $n=20 ; \mathrm{HT}, n=14$ ) relative to the control diet (WT, $n=18 ; \mathrm{HT}, n=16$ ). (c) At specific time points, PDE11A KO and PDE11A HT mice chronically fed the $0.4 \%$ lithium chow demonstrated a significant anti-manic-like attenuation of the amphetamine-stimulated rise in horizontal beam breaks relative to WT littermates (WT and HT, $n=26 ; \mathrm{KO}, n=25)$. Post hoc, "vs control chow, $P=0.011$; *vs KO, $P=0.015-0.002 ;{ }^{\circledR}$ vs HT, $P=0.02$. Data passed normality and equal variance and are graphed as means \pm s.e.m. Brightness and contrast adjusted for graphical clarity of autoradiographic images. HT, heterozygous; KO, knockout; PDE11A, phosphodiesterase $11 \mathrm{~A}$.

Figures S1-S3), and behavioral experiments using Pde11a KO mice show decreasing PDE11A expression is sufficient to increase lithium responsivity (Figure 4). A key driver of the PDE11A4 expression differences noted between mouse strains is a nonsynonymous coding SNP falling within the PDE11A4 GAF-B domain, a mutation that alters levels of PDE11A4 homodimerization and compartmentalization (Figure 3). Our findings are consistent with a number of studies examining tissue from patients with bipolar disorder that describe significant changes in the CAMP cascade, ${ }^{18-26,66,67}$ including changes restricted to specific subcellular compartments ${ }^{18,23,25}$ and changes associated with lithium responsivity. ${ }^{24,68}$

Strain differences in PDE11A4 compartmentalization are associated with genetically-driven differences in PDE11A4 homodimerization

We show that homodimerization is a key regulator of PDE11A4 subcellular compartmentalization (Figure 3). It has long been known that all PDEs homodimerize/oligomerize, but the functional significance of this interaction remained unknown. ${ }^{69}$ Homodimerization of PDE11A4 does not alter its substrate affinity; ${ }^{58}$ instead, it appears to control which pool of cyclic nucleotides PDE11A4 can degrade. We believe homodimerization/oligomerization will similarly control the subcellular compartmentalization of other GAF domain-containing PDE families, given recent reports showing GAF-B mutations change the subcellular compartmentalization of PDE6C (effect on oligomerization not directly assessed). ${ }^{70}$

A non-synonymous coding SNP falling within the GAF-B homodimerization domain encodes an alanine at amino acid 499 in $\mathrm{C} 57 \mathrm{BL} / 6 \mathrm{~J}$ mice but a threonine in BALB/CJ mice. In vitro experiments show changing amino acid 499 from an alanine (499A) to a threonine (499T) or a phosphomimic aspartate (499D) promotes homodimerization (Figures $3 \mathrm{~d}$ and $\mathrm{f}$ ) and increases trafficking of PDE11A4 into aggregates (Figures $3 \mathrm{~h}$ and i). Furthermore, 499T/D shifts PDE11A4 from the cytosol to the membrane (Figures $3 \mathrm{I}$ and $\mathrm{n}$ ), perfectly replicating in vivo PDE11A4 expression observed in VHIPP of BALB/CJ vs C57BL/6J mice (Figure 2c). In contrast, disrupting homodimerization using an isolated GAF-B domain (Figures $3 e$ and g) decreases trafficking of PDE11A4 into aggregates (Figure 3j), shifts PDE11A4 from the membrane to the cytosol (Figures $3 \mathrm{~m}$ and o), and blocks the effect of the 499T and 499D mutations (Figure 3k). Indeed, 

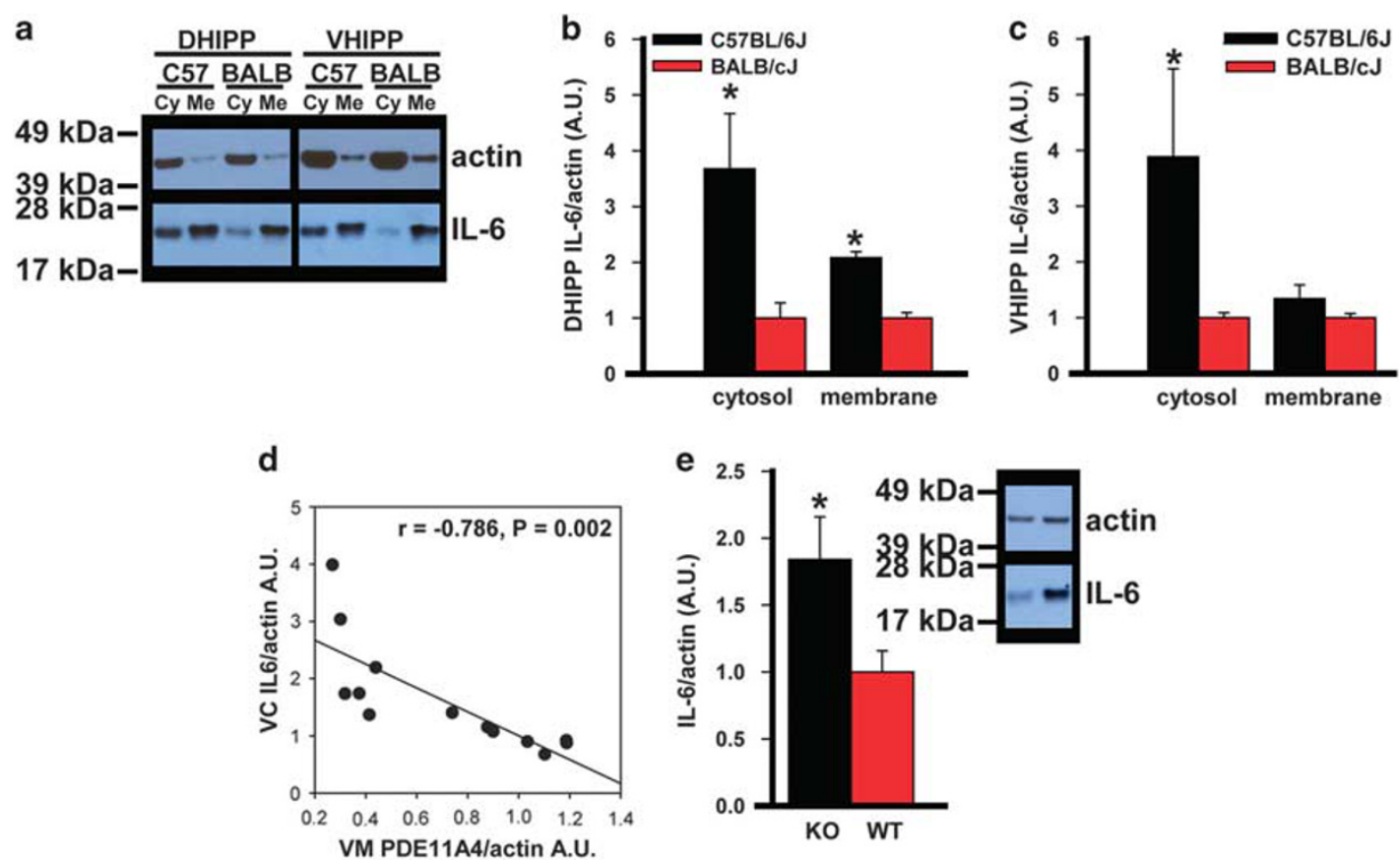

Figure 5. Decreasing PDE11A expression increases IL-6 expression. To determine whether decreased PDE11A4 may drive expression of pathophysiological markers, we examined expression of the pro-inflammatory cytokine IL-6. (a) Western blots show that (b) C57BL/6J mice express more IL- 6 relative to BALB/cJ mice in both the cytosolic and membrane fractions of DHIPP $(n=6$ per strain for membrane and 8 per strain for cytosol). (c) In VHIPP, however, C57BL/6J mice only express more IL-6 relative to BALB/cJ mice in the cytosolic fraction ( $n=8$ per strain per compartment). (d) Interestingly, PDE11A4 expression in the VHIPP membrane (VM) fraction correlates negatively with IL-6 expression in the VHIPP cytosol (VC), such that C57BL/6J and BALB/cJ mice with lower PDE11A4 expression exhibit higher levels of IL-6 expression. (e) Decreasing PDE11A expression appears sufficient to upregulate IL-6 because PDE11A KO mice demonstrate significantly more IL-6 expression relative to paired sex-matched WT littermates in DHIPP (WT, $n=17 ; \mathrm{KO}, n=19)$. Data in panel $\mathbf{b}$ passed normality and equal variance. Data in panel $\mathbf{c}$ and e failed normality; therefore, nonparametric tests were used for those data sets. Data are graphed as means \pm s.e.m. Brightness and contrast adjusted for graphical clarity of blot images. Post hoc, *vs BALB/cJ or WT, $P \leq 0.005-0.001$. DHIPP, dorsal hippocampus; IL-6, interleukin-6; KO, knockout; PDE11A, phosphodiesterase 11A; VHIPP, ventral hippocampus.

homodimerization appears to regulate the subcellular compartmentalization of several proteins, including extracellular signalregulated kinase $2,{ }^{71} \mathrm{PleD}^{72}$ and Islet-Brain $1 .^{73}$ Differences in protein translation efficiency may also contribute to strain differences in PDE11A4 protein expression as it is a major factor controlling the relative protein expression levels of PDE6 subunits in the retina. ${ }^{74}$

PDE11A4 expression and compartmentalization differ between DHIPP and VHIPP

We and others have shown in multiple mouse and rat strains that Pde11a4 mRNA and protein are enriched in the hippocampus, with expression that is substantially higher in VHIPP vs DHIPP. $4-6,42,75,76$ Here we believe we noted for the first time a mouse strain (that is, BALB/cJ) in which Pde11a4 mRNA expression in DHIPP was equivalent to that in VHIPP (Figure 1a, Supplementary Figure S1). Even though PDE11A4 mRNA expression did not differ between DHIPP and VHIPP in the BALB/CJ, PDE11A4 protein did show its typical dorsal-ventral gradient at the protein level in this mouse strain (Figures 1d and e, Supplementary Figure S2C-D). The molecular mechanisms driving these dorsal-ventral gradients in mRNA and protein expression remain to be determined. Preliminary evidence from our laboratory suggests the dorsal-ventral gradient in mRNA expression seen in most mouse and rat strains characterized to date is driven by transcriptional regulation of the promotor, as demonstrated by differential DHIPP vs VHIPP expression of an mCherry-reporter construct driven by the C57BL/6J PDE11A4 promoter (MP Kelly, personal observations).
Indeed, Sox-5-a putative PDE11A4 transcription factor ${ }^{3}$-is expressed at significantly higher levels in VHIPP vs DHIPP. ${ }^{75}$

There is also a distinction between DHIPP vs VHIPP in terms of the subcellular compartments that show PDE11A4 protein expression differences between the mouse strains. In DHIPP, PDE11A4 expression differs between the strains in both cytosolic and membrane compartments, but in VHIPP PDE11A4 expression differs between $\mathrm{C} 57 \mathrm{BL} / 6 \mathrm{~J}$ and $\mathrm{BALB} / \mathrm{CJ}$ mice only in the membrane compartment. Again, the mechanism for this remains to be determined, but we hypothesize that phosphorylation of 499T in the BALB/cJ may make PDE11A4 more or less susceptible to other post-translational modifications whose key modifying enzymes show differential expression along the DHIPP-VHIPP axis. Indeed, preliminary evidence from our lab shows that phosphorylation of one site (for example, S162) within the PDE11A4 N-terminal domain affects the phosphorylation of other N-terminal sites (for example, S117 and S124) as well as the functional consequences of those phosphorylation events (WR Capell and MP Kelly, personal observations). Interestingly, protein kinase $C$ is expressed at much higher levels in DHIPP vs VHIPP ${ }^{75}$ and is predicted to phosphorylate PDE11A4 at the aforementioned $\mathrm{N}$-terminal residues. ${ }^{5}$ Exploring this hypothesis will be of interest to future studies.

Pde11a deletion is sufficient to increase lithium responsivity Pde11a KO mice were more sensitive than WT mice to the effects of lithium in both TST and amphetamine-stimulated hyperactivity. This is consistent with the fact that the C57BL/6J and 129S6/ SvEvTac mice with low PDE11A4 expression are more sensitive to 
the effects of lithium in TST and forced swim test (FST) than BALB/cJ mice with high PDE11A4 expression (Figures 1 and 2, Supplementary Figure S1-S3). ${ }^{46,53}$ Specifically, following 3 weeks of lithium treatment, C57BL/6J mice demonstrated a significant antidepressant-like effect in both TST and FST, 129S6/SvEvTac mice demonstrated a significant antidepressant-like effect only in FST, and BALB/CJ mice demonstrated no effect in either assay. ${ }^{46}$ Similarly, 129S6/SvEvTac mice showed a significant antidepressant-like effect of acute lithium treatment in TST and FST, C57BL/6J mice showed a limited antidepressant-like effect in FST, and BALB/CJ mice showed no effect in either assay. C57BL/6J mice have also shown a significant anti-manic-like effect of lithium in amphetamine-stimulated locomotor activity; whereas, $12956 /$ SvEvTac mice did not (BALB/cJ mice not tested). ${ }^{63}$ The differential lithium sensitivity of $\mathrm{C} 57 \mathrm{BL} / 6 \mathrm{~J}$ vs $\mathrm{BALB} / \mathrm{CJ}$ mice is observed in both males and females whether reared by their own parents or cross-fostered by the opposite strain, suggesting a genetic difference in the offspring is driving this phenotype as opposed to strain differences in maternal care. ${ }^{53}$ Our studies here suggest that differential expression and compartmentalization of PDE11A4 may be at least 1 of those mechanisms.

It is important to note that the differential lithium sensitivity of the Pde11a KOs in TST was only detected in group-housed mice (Figure 4b). Specifically, both Pde11a WT and KO mice demonstrated antidepressant-like effects of lithium in TST when single housed, but only Pde11a KO mice demonstrated this antidepressant-like effect of lithium when group housed. This may suggest that, even when group housed, Pde11a KO mice experience subjective or objective social isolation, making them susceptible to the behavioral effects of lithium in TST. Alternatively, social isolation may decrease PDE11A4 expression in the WTs, making them susceptible to the effects of lithium in TST. Indeed, recent studies from our laboratory show that a normally social C57BL/6J mouse prefers to interact with a PDE11A WT vs. its $\mathrm{KO}$ littermate, and that social isolation decreases PDE11A4 protein expression specifically within the membrane compartment of the VHIPP. ${ }^{65}$ In this context it is interesting to note that lithium increases social interactions in FMR1 KO mice and their WT counterparts. ${ }^{77}$ It will be of interest to future studies to further characterize the effects of lithium in the Pde11a KO mice in other antidepressant-predictive assays (for example, FST ${ }^{46,53}$ ) or in the context of manipulations intended to model aspects of bipolar disorder (for example, amphetamine sensitization ${ }^{41}$ ), particularly give the limited effect size observed herein in TST.

\section{Reduced PDE11A4 expression may reflect a lithium-sensitive pathophysiology}

Lower levels of PDE11A4 may predict better lithium responsiveness either because (1) a state of decreased PDE11A4 expression represents a specific disease state that is most responsive to lithium or (2) because reducing PDE11A4 expression somehow boosts lithium's effects. PDE11A KO mice exhibit a number of behavioral, anatomical, and biochemical phenotypes relevant to psychiatric disease. ${ }^{6}$ Previously, we reported that PDE11A KO mice exhibit significantly reduced CAMP-PDE activity in VHIPP. ${ }^{6}$ We have struggled to measure changes in CAMP at the level of the whole VHIPP, ${ }^{6}$ we believe due to a signal:noise issue caused by the sparse nature of PDE11A4 expression within the hippocampus (that is, it is only expressed in a subpopulation of neurons in CA1 and subiculum, not CA3 nor DG). ${ }^{61}$ Interestingly, elevated levels of pCREB have been found in lymphoblasts from lithium-responsive bipolar disorder patients and their relatives compared to healthy controls. $^{24}$ Here we were not able to measure a significant increase in PCREB in the PDE11A KO mice when examining the VHIPP as a whole; however, we were able to detect a significant decrease in Arhgap32 mRNA (a.k.a. p250gap, RICS) when examining ventral CA1 specifically (Figure 4a). Arhgap32 is a gene that is downregulated by increased pCREB signaling via miR132. ${ }^{51,52}$ Of interest, Arhgap32 controls dendritic plasticity and has been genetically linked to the ability to identify/describe moods and feeling, schizophrenia, and autism. ${ }^{78-80}$ Increased activation of the cAMP cascade may represent a specific pathophysiology that is particularly responsive to lithium treatment because chronic lithium appears to decrease PCREB in hippocampus and cortex of rats. $^{81}$

Here, C57BL/6J and PDE11A KO mice also exhibit significantly higher levels of the pro-inflammatory cytokine IL- 6 relative to $\mathrm{BALB} / \mathrm{CJ}$ and WT mice, respectively (Figure 5). Increased IL-6 and/ or IL-6 receptor expression is not selectively associated with bipolar disorder but has been repeatedly observed in patients with mania, depression, and suicidal ideation, ${ }^{30-34,36,37}$ and lithium decreases IL-6 expression in patient tissue. ${ }^{35}$ In addition, PDE11A $\mathrm{KO}$ mice and iPSC-derived hippocampal neurons from bipolar patients exhibit altered signaling in the neuroactive-ligand receptor pathway and increased neural activity). ${ }^{6,16,65}$ Altogether, these data argue that a state of decreased PDE11A4 expression represents a lithium-responsive pathophysiology. That said, lithium decreases PDE11A mRNA expression in iPSC-derived hippocampal neurons from lithium-responsive patients but not non-responsive patients, which is consistent with the idea that lowering PDE11A is key to the therapeutic actions of lithium. ${ }^{16}$ One possible explanation for these dichotomous results is the fact that the iPSC-derived hippocampal neurons were described as dentate gyrus granule cell-like neurons; however, endogenous PDE11A4 in the rodent brain is expressed in CA1 and subiculum, but not DG. Thus, it will be critical for future studies to determine which, if any, isoform of PDE11A is expressed in vivo in the human dentate gyrus, what functional implications that would hold for the hippocampal circuit, and exactly why lowering PDE11A expression is sufficient to increase lithium responsivity.

\section{CONFLICT OF INTEREST}

MPK received consulting fees from ASUBIO, and Deallus for projects unrelated to the current manuscript and is a former employee of Pfizer. MJA is an employee of Pfizer. $\Pi$ is a former employee of Pfizer and is currently an employee of Charles River and Biogen. The remaining authors declare no conflict of interest.

\section{ACKNOWLEDGMENTS}

We thank Jennifer Klett for technical assistance and Drs John Kelsoe and Susan Leckband for helpful discussion. This research was supported by a Research Starter Grant in Pharmacology \& Toxicology from the PhRMA Foundation, an ASPIRE award from the Office of the Vice President for Research from the University of South Carolina, a Research Development Fund Award from the University of South Carolina School of Medicine, 1R01MH101130 from NIMH, and a NARSAD Young Investigator Award from the Brain \& Behavior Research Foundation (all awards to MPK).

\section{REFERENCES}

1 Malhi GS, Tanious M, Das P, Berk M. The science and practice of lithium therapy. Aust N Z J Psychiatry 2012; 46: 192-211.

2 Yuasa K, Ohgaru T, Asahina M, Omori K. Identification of rat cyclic nucleotide phosphodiesterase 11A (PDE11A): comparison of rat and human PDE11A splicing variants. Eur J Biochem 2001; 268: 4440-4448.

3 Yuasa K, Kanoh Y, Okumura K, Omori K. Genomic organization of the human phosphodiesterase PDE11A gene. Evolutionary relatedness with other PDEs containing GAF domains. Eur J Biochem 2001; 268: 168-178.

4 Kelly MP, Adamowicz W, Bove S, Hartman AJ, Mariga A, Pathak G et al. Select 3',5'-cyclic nucleotide phosphodiesterases exhibit altered expression in the aged rodent brain. Cell Signal 2014; 26: 383-397.

5 Kelly MP. Does phosphodiesterase 11A (PDE11A) hold promise as a future therapeutic target? Curr Pharm Des 2015; 21: 389-416.

6 Kelly MP, Logue SF, Brennan J, Day JP, Lakkaraju S, Jiang L et al. Phosphodiesterase $11 \mathrm{~A}$ in brain is enriched in ventral hippocampus and deletion causes psychiatric disease-related phenotypes. Proc Natl Acad Sci USA 2010; 107: 8457-8462. 
7 Wong ML, Whelan F, Deloukas P, Whittaker P, Delgado M, Cantor RM et al. Phosphodiesterase genes are associated with susceptibility to major depression and antidepressant treatment response. Proc Natl Acad Sci USA 2006; 103: 15124-15129.

8 Luo HR, Wu GS, Dong C, Arcos-Burgos M, Ribeiro L, Licinio J et al. Association of PDE11A global haplotype with major depression and antidepressant drug response. Neuropsychiatr Dis Treat 2009; 5: 163-170.

9 Cabanero M, Laje G, Detera-Wadleigh S, McMahon FJ. Association study of phosphodiesterase genes in the Sequenced Treatment Alternatives to Relieve Depression sample. Pharmacogenet Genomics 2009; 19: 235-238.

10 Couzin J. Science and commerce. Gene tests for psychiatric risk polarize researchers. Science 2008; 319: 274-277.

11 Kelsoe J. Method to Predict Response to Treatment for Psychiatric Illnesses. In: Office UPT. The Regents of The University of California: Oakland, CA, USA, 2010, pp 1.

12 Coon $\mathrm{H}$, Darlington T, Pimentel R, Smith KR, Huff CD, Hu H et al. Genetic risk factors in two Utah pedigrees at high risk for suicide. Transl Psychiatry 2013; 3: e325.

13 Laje G, Perlis RH, Rush AJ, McMahon FJ. Pharmacogenetics studies in STAR*D: strengths, limitations, and results. Psychiatr Serv 2009; 60: 1446-1457.

14 Perlis RH, Fijal B, Dharia S, Heinloth AN, Houston JP. Failure to replicate genetic associations with antidepressant treatment response in duloxetine-treated patients. Biol Psychiatry 2010; 67: 1110-1113.

15 Malhi GS, Tanious M, Das P, Coulston CM, Berk M. Potential mechanisms of action of lithium in bipolar disorder. Current understanding. CNS Drugs 2013; 27: 135-153.

16 Mertens J, Wang QW, Kim Y, Yu DX, Pham S, Yang B et al. Differential responses to lithium in hyperexcitable neurons from patients with bipolar disorder. Nature 2015; 527: 95-99.

17 Gould TD, Quiroz JA, Singh J, Zarate CA, Manji HK. Emerging experimental therapeutics for bipolar disorder: insights from the molecular and cellular actions of current mood stabilizers. Mol Psychiatry 2004; 9: 734-755.

18 Rahman S, Li PP, Young LT, Kofman O, Kish SJ, Warsh JJ. Reduced [3H]cyclic AMP binding in postmortem brain from subjects with bipolar affective disorder. J Neurochem 1997; 68: 297-304.

19 Avissar S, Nechamkin Y, Barki-Harrington L, Roitman G, Schreiber G. Differential G protein measures in mononuclear leukocytes of patients with bipolar mood disorder are state dependent. J Affect Disord 1997; 43: 85-93.

20 Avissar S, Schreiber G. The involvement of $G$ proteins and regulators of receptor-G protein coupling in the pathophysiology, diagnosis and treatment of mood disorders. [Review] [109 refs]. Clin Chim Acta 2006; 366: 37-47.

21 Schreiber G, Avissar S. Lithium sensitive G protein hyperfunction: a dynamic model for the pathogenesis of bipolar affective disorder. Med Hypotheses 1991; 35: 237-243.

22 Schreiber G, Avissar S, Danon A, Belmaker RH. Hyperfunctional G proteins in mononuclear leukocytes of patients with mania. Biological Psychiatry 1991; 29: 273-280.

23 Fields A, Li PP, Kish SJ, Warsh JJ. Increased cyclic AMP-dependent protein kinase activity in postmortem brain from patients with bipolar affective disorder. J Neurochem 1999; 73: 1704-1710.

24 Alda M, Shao L, Wang JF, Lopez de Lara C, Jaitovich-Groisman I, Lebel V et al. Alterations in phosphorylated cAMP response element-binding protein (pCREB) signaling: an endophenotype of lithium-responsive bipolar disorder? Bipolar Disord 2013; 15: 824-831.

25 Chang A, Li PP, Warsh JJ. Altered CAMP-dependent protein kinase subunit immunolabeling in post-mortem brain from patients with bipolar affective disorder. J Neurochem 2003; 84: 781-791.

26 Dowlatshahi D, MacQueen GM, Wang JF, Reiach JS, Young LT. G. Protein-coupled cyclic AMP signaling in postmortem brain of subjects with mood disorders: effects of diagnosis, suicide, and treatment at the time of death. J Neurochem 1999; 73: 1121-1126.

27 Mori S, Tardito D, Dorigo A, Zanardi R, Smeraldi E, Racagni G et al. Effects of lithium on CAMP-dependent protein kinase in rat brain. Neuropsychopharmacology 1998; 19: 233-240.

28 Jensen JB, Mork A. Altered protein phosphorylation in the rat brain following chronic lithium and carbamazepine treatments. Eur Neuropsychopharmacol 1997; 7: 173-179.

29 Casebolt TL, Jope RS. Effects of chronic lithium treatment on protein kinase $C$ and cyclic AMP-dependent protein phosphorylation. Biol Psychiatry 1991; 29: 233-243.

30 Dowlati Y, Herrmann N, Swardfager W, Liu H, Sham L, Reim EK et al. A meta-analysis of cytokines in major depression. Biol Psychiatry 2010; 67: 446-457.

31 Maes M, Bosmans E, De Jongh R, Kenis G, Vandoolaeghe E, Neels H. Increased serum IL-6 and IL-1 receptor antagonist concentrations in major depression and treatment resistant depression. Cytokine 1997; 9: 853-858.
32 Maes M, Bosmans E, Calabrese J, Smith R, Meltzer HY. Interleukin-2 and interleukin- 6 in schizophrenia and mania: effects of neuroleptics and mood stabilizers. J Psychiatr Res 1995; 29: 141-152.

33 Brietzke E, Stertz L, Fernandes BS, Kauer-Sant'anna M, Mascarenhas M, Escosteguy Vargas $A$ et al. Comparison of cytokine levels in depressed, manic and euthymic patients with bipolar disorder. J Affect Disord 2009; 116: 214-217.

34 Simon NM, McNamara K, Chow CW, Maser RS, Papakostas Gl, Pollack MH et al. A detailed examination of cytokine abnormalities in Major Depressive Disorder. Eur Neuropsychopharmacol 2008; 18: 230-233.

35 Watanabe S, Iga J, Nishi A, Numata S, Kinoshita M, Kikuchi K et al. Microarray analysis of global gene expression in leukocytes following lithium treatment. Hum Psychopharmacol 2014; 29: 190-198.

36 Niculescu AB, Levey DF, Phalen PL, Le-Niculescu H, Dainton HD, Jain N et al. Understanding and predicting suicidality using a combined genomic and clinical risk assessment approach. Mol Psychiatry 2015; 20: 1266-1285.

37 Khandaker GM, Pearson RM, Zammit S, Lewis G, Jones PB. Association of serum interleukin 6 and C-reactive protein in childhood with depression and psychosis in young adult life: a population-based longitudinal study. JAMA Psychiatry 2014; 71: $1121-1128$.

38 Stephenson DT, Coskran TM, Kelly MP, Kleiman RJ, Morton D, O'Neill SM et al. The distribution of phosphodiesterase $2 A$ in the rat brain. Neuroscience 2012; 226: 145-155.

39 Charych El, Jiang LX, Lo F, Sullivan K, Brandon NJ. Interplay of palmitoylation and phosphorylation in the trafficking and localization of phosphodiesterase 10A: implications for the treatment of schizophrenia. J Neurosci 2010; 30: 9027-9037.

40 Sukoff Rizzo SJ, Neal SJ, Hughes ZA, Beyna M, Rosenzweig-Lipson S, Moss SJ et al. Evidence for sustained elevation of IL- 6 in the CNS as a key contributor of depressive-like phenotypes. Transl Psychiatry 2012; 2: e199.

41 Pathak G, Ibrahim BA, McCarthy SA, Baker K, Kelly MP. Amphetamine sensitization in mice is sufficient to produce both manic- and depressive-related behaviors as well as changes in the functional connectivity of corticolimbic structures. Neuropharmacology 2015; 95: 434-447.

42 Kelly MP. Putting together the pieces of phosphodiesterase distribution patterns in the brain: A jigsaw puzzle of cyclic nucleotide regulation In: Brandon NJ, West AR (eds). Cyclic Nucleotide Phosphodiesterases in the Central Nervous System: From Biology to Disease. John Wiley \& Sons, Inc.: NJ, USA, 2014.

43 Jackson RJ, Howell MT, Kaminski A. The novel mechanism of initiation of picornavirus RNA translation. Trends Biochem Sci 1990; 15: 477-483.

44 Shaner NC, Campbell RE, Steinbach PA, Giepmans BN, Palmer AE, Tsien RY Improved monomeric red, orange and yellow fluorescent proteins derived from Discosoma sp. red fluorescent protein. Nat Biotechnol 2004; 22: 1567-1572.

45 Blount MA, Beasley A, Zoraghi R, Sekhar KR, Bessay EP, Francis SH et al. Binding of tritiated sildenafil, tadalafil, or vardenafil to the phosphodiesterase-5 catalytic site displays potency, specificity, heterogeneity, and cGMP stimulation. Mol Pharmacol 2004; 66: 144-152.

46 Can A, Blackwell RA, Piantadosi SC, Dao DT, O'Donnell KC, Gould TD. Antidepressant-like responses to lithium in genetically diverse mouse strains. Genes Brain Behav 2011; 10: 434-443.

47 Kelly MP, Logue SF, Dwyer JM, Beyer CE, Majchrowski H, Cai Z et al. The supraadditive hyperactivity caused by an amphetamine-chlordiazepoxide mixture exhibits an inverted- $U$ dose response: negative implications for the use of a model in screening for mood stabilizers. Pharmacol Biochem Behav 2009; 92 649-654.

48 Hughes ZA, Liu F, Platt BJ, Dwyer JM, Pulicicchio CM, Zhang G et al. WAY-200070, a selective agonist of estrogen receptor beta as a potential novel anxiolytic/ antidepressant agent. Neuropharmacology 2008; 54: 1136-1142.

49 Malberg JE, Platt B, Rizzo SJ, Ring RH, Lucki I, Schechter LE et al. Increasing the levels of insulin-like growth factor-I by an IGF binding protein inhibitor produces anxiolytic and antidepressant-like effects. Neuropsychopharmacology 2007; 32: 2360-2368.

50 Kelly MP, Cheung YF, Favilla C, Siegel SJ, Kanes SJ, Houslay MD et al. Constitutive activation of the G-protein subunit Galphas within forebrain neurons causes PKA-dependent alterations in fear conditioning and cortical Arc mRNA expression. Learn Mem 2008; 15: 75-83.

51 Wayman GA, Davare M, Ando H, Fortin D, Varlamova O, Cheng HY et al. An activity-regulated microRNA controls dendritic plasticity by down-regulating p250GAP. Proc Natl Acad Sci USA 2008; 105: 9093-9098.

52 Dhar M, Zhu M, Impey S, Lambert TJ, Bland T, Karatsoreos IN et al. Leptin induces hippocampal synaptogenesis via CREB-regulated microRNA-132 suppression of p250GAP. Mol Endocrinol 2014; 28: 1073-1087.

53 Can A, Piantadosi SC, Gould TD. Differential antidepressant-like response to lithium treatment between mouse strains: effects of sex, maternal care, and mixed genetic background. Psychopharmacology (Berl) 2013; 228: 411-418.

54 Karolchik D, Barber GP, Casper J, Clawson H, Cline MS, Diekhans M et al. The UCSC Genome Browser database: 2014 update. Nucleic Acids Res 2014; 42: D764-D770. 
55 Bogue MA, Peters LL, Paigen B, Korstanje R, Yuan R, Ackert-Bicknell $C$ et al. Accessing data resources in the mouse phenome database for genetic analysis of murine life span and health span. J Gerontol A Biol Sci Med Sci 2016; 71: 170-177.

56 Sherry ST, Ward MH, Kholodov M, Baker J, Phan L, Smigielski EM et al. dbSNP: the NCBI database of genetic variation. Nucleic Acids Res 2001; 29: 308-311.

57 Chaney JL, Clark PL. Roles for synonymous codon usage in protein biogenesis. Annu Rev Biophys 2015; 44: 143-166.

58 Weeks JL 2nd, Zoraghi R, Francis SH, Corbin JD. N-Terminal domain of phosphodiesterase-11A4 (PDE11A4) decreases affinity of the catalytic site for substrates and tadalafil, and is involved in oligomerization. Biochemistry 2007; 46: 10353-10364.

59 Edwards HV, Christian F, Baillie GS. cAMP: novel concepts in compartmentalised signalling. Semin Cell Dev Biol 2012; 23: 181-190.

60 McCahill AC, Huston E, Li X, Houslay MD. PDE4 associates with different scaffolding proteins: modulating interactions as treatment for certain diseases. Handb Exp Pharmacol 2008; 125-166.

61 Hegde S, Capell WR, Ibrahim BA, Klett J, Patel NS, Sougiannis AT et al. Phosphodiesterase 11A (PDE11A), enriched in ventral hippocampus neurons, is required for consolidation of social but not nonsocial memories in mice. Neuropsychopharmacology, 2016; E-pub ahead of print; doi:10.1038/npp.2016.106.

62 Steru L, Chermat R, Thierry B, Simon P. The tail suspension test: a new method for screening antidepressants in mice. Psychopharmacology (Berl) 1985; 85: 367-370.

63 Gould TD, O'Donnell KC, Picchini AM, Manji HK. Strain differences in lithium attenuation of $\mathrm{d}$-amphetamine-induced hyperlocomotion: a mouse model for the genetics of clinical response to lithium. Neuropsychopharmacology 2007; 32: 1321-1333.

64 Gould TJ, Keith RA, Bhat RV. Differential sensitivity to lithium's reversal of amphetamine-induced open-field activity in two inbred strains of mice. Behav Brain Res 2001; 118: 95-105.

65 Hegde S, Ji H, Oliver D, Patel NS, Poupore N, Shtutman M et al. PDE11A regulates social behaviors and is a key mechanism by which social experience sculpts the brain. Neuroscience, 2016; E-pub ahead of print; doi:10.1016/j. neuroscience.2016.08.019.

66 Fatemi SH, Folsom TD, Reutiman TJ, Vazquez G. Phosphodiesterase signaling system is disrupted in the cerebella of subjects with schizophrenia, bipolar disorder, and major depression. Schizophr Res 2010; 119: 266-267.

67 Fatemi SH, Reutiman TJ, Folsom TD, Lee S. Phosphodiesterase-4A expression is reduced in cerebella of patients with bipolar disorder. Psychiatr Genet 2008; 18: 282-288.

68 Sun X, Young LT, Wang JF, Grof P, Turecki G, Rouleau GA et al. Identification of lithium-regulated genes in cultured lymphoblasts of lithium responsive subjects with bipolar disorder. Neuropsychopharmacology 2004; 29: 799-804.

69 Francis SH, Blount MA, Corbin JD. Mammalian cyclic nucleotide phosphodiesterases: molecular mechanisms and physiological functions. Physiol Rev 2011; 91: 651-690.
70 Cheguru P, Majumder A, Artemyev NO. Distinct patterns of compartmentalization and proteolytic stability of PDE6C mutants linked to achromatopsia. Mol Cell Neurosci 2015; 64: 1-8.

71 Khokhlatchev AV, Canagarajah B, Wilsbacher J, Robinson M, Atkinson M, Goldsmith $\mathrm{E}$ et al. Phosphorylation of the MAP kinase ERK2 promotes its homodimerization and nuclear translocation. Cell 1998; 93: 605-615.

72 Paul R, Abel S, Wassmann P, Beck A, Heerklotz H, Jenal U. Activation of the diguanylate cyclase PleD by phosphorylation-mediated dimerization. J Biol Chem 2007; 282: 29170-29177.

73 Borsello T, Centeno C, Riederer IM, Haefliger JA, Riederer BM. Phosphorylationdependent dimerization and subcellular localization of islet-brain 1/c-Jun N-terminal kinase-interacting protein 1. J Neurosci Res 2007; 85: 3632-3641.

74 Piri N, Yamashita CK, Shih J, Akhmedov NB, Farber DB. Differential expression of rod photoreceptor CGMP-phosphodiesterase alpha and beta subunits: mRNA and protein levels. J Biol Chem 2003; 278: 36999-37005.

75 Cembrowski MS, Bachman JL, Wang L, Sugino K, Shields BC, Spruston N. Spatial Gene-Expression Gradients Underlie Prominent Heterogeneity of CA1 Pyramidal Neurons. Neuron 2016; 89: 351-368.

76 Jager R, Russwurm C, Schwede F, Genieser HG, Koesling D, Russwurm M. Activation of PDE10 and PDE11 phosphodiesterases. J Biol Chem 2012; 287: 1210-1219.

77 Liu ZH, Chuang DM, Smith CB. Lithium ameliorates phenotypic deficits in a mouse model of fragile $X$ syndrome. Int J Neuropsychopharmacol 2011; 14: 618-630.

78 Ohi K, Hashimoto R, Nakazawa T, Okada T, Yasuda Y, Yamamori H et al. The p250GAP gene is associated with risk for schizophrenia and schizotypal personality traits. PLoS One 2012; 7: e35696.

79 Akshoomoff N, Mattson SN, Grossfeld PD. Evidence for autism spectrum disorder in Jacobsen syndrome: identification of a candidate gene in distal 11q. Genet Med 2015; 17: 143-148.

80 Mezzavilla M, Ulivi S, Bianca ML, Carlino D, Gasparini P, Robino A. Analysis of functional variants reveals new candidate genes associated with alexithymia. Psychiatry Res 2015; 227: 363-365.

81 Chen B, Wang JF, Hill BC, Young LT. Lithium and valproate differentially regulate brain regional expression of phosphorylated CREB and c-Fos. Brain Res Mol Brain Res 1999; 70: 45-53.

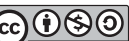

This work is licensed under a Creative Commons AttributionNonCommercial-ShareAlike 4.0 International License. The images or other third party material in this article are included in the article's Creative Commons license, unless indicated otherwise in the credit line; if the material is not included under the Creative Commons license, users will need to obtain permission from the license holder to reproduce the material. To view a copy of this license, visit http:// creativecommons.org/licenses/by-nc-sa/4.0/

(c) The Author(s) 2017

Supplementary Information accompanies the paper on the Molecular Psychiatry website (http://www.nature.com/mp) 